\title{
Thyroid Fine-Needle Aspiration Practice in the Philippines
}

\author{
Agustina D. Abelardo \\ Department of Pathology, College of Medicine, \\ University of the Philippines Manila, Manila, \\ Philippines
}

\author{
Received: July 4, 2017 \\ Accepted: July 14, 2017 \\ Corresponding Author \\ Agustina D. Abelardo, MD, MIAC \\ Department of Pathology, College of Medicine, \\ University of the Philippines Manila, 547 Pedro Gil \\ St Ermita, Manila 1000, Philippines \\ Tel: +63-2-526-45-50 \\ Fax: +63-2-526-45-50 \\ E-mail: abelardoagustina@yahoo.com
}

\begin{abstract}
Fine-needle aspiration (FNA) is a well accepted initial approach in the management of thyroid lesions. It has come a long way since its introduction for nearly a century ago. In the Philippines, FNA of the thyroid was first introduced 30 years ago and has been utilized until now as a mainstay in the diagnosis of thyroid malignancy. The procedure is performed by pathologists, endocrinologists, surgeons, and radiologists. Most pathologists report the cytodiagnosis using a combination of the aspiration biopsy cytology method that closely resembles the histopathologic diagnosis of thyroid disorders and the six-tier nomenclature of The Bethesda System for Reporting Thyroid Cytopathology. Local endocrinologists and surgeons follow the guidelines of the 2015 American Thyroid Association in the management of thyroid disorders. There is still a paucity of local research studies but available data deal with cytohistologic correlations, sensitivity, specificity, and accuracy rates as well as usefulness of ultrasound-guided FNA. Cytohistologic correlations have a wide range of sensitivity from $30.7 \%$ to $73 \%$ and specificity from $83 \%$ to $100 \%$. The low sensitivity can be attributed to poor tissue sampling since a majority of the thyroid FNA is done by palpation only. The reliability can be improved if FNA is guided by ultrasound as attested in both international and local studies. Overall, FNA of the thyroid has enabled the diagnosis of thyroid disorders with an accuracy of $72.8 \%$ to $87.2 \%$ and it correlates well with histopathology.
\end{abstract}

Key Words: Fine-needle aspiration cytology; Thyroid fine-needle aspiration; The Bethesda System for Reporting Thyroid Cytopathology (TBSRTC)
Fine-needle aspiration (FNA) biopsy has proved to be an accurate, safe, and cost-effective method in the initial management of thyroid nodules. It is a widely accepted diagnostic tool all over the world with a history spanning from the reports of Mannheim followed by the works of Martin \& Ellis on needle aspirates way back in the 1930 s. ${ }^{1-3}$ In the 1950 s to the 1970 s, Scandinavian pioneers from Sweden continued to develop the method including aspiration biopsy cytology of practically all organs. ${ }^{4-6}$ To date, voluminous works on accuracy data, cytohistologic correlations, the usefulness of six-tier Bethesda System for Reporting Thyroid Cytopathology (TBSRTC), and a wide array of ancillary studies on thyroid FNA are available.

\section{HISTORY OF FINE-NEEDLE ASPIRATION IN THE PHILIPPINES}

FNA of the thyroid started in the late 1980s at the Philippine General Hospital, a 1,500-bed tertiary government hospital that serves as the training facility for health sciences students enrolled in the University of the Philippines. Consultants and resident physician trainees from the departments of pathology and surgery started doing FNA of various palpable lesions mainly in the neck, $40 \%$ to $50 \%$ of which were thyroid aspirates. Thereafter, an aspiration cytology unit in the Department of Pathology housed at the College of Medicine of the University was established upon the return of a faculty member of the department from a 2-month training in FNA at the Cytology Unit of Karolinska Hospital in Stockholm, Sweden, in 1987. Two years later, another pathologist with interest in cytopathology established an FNA clinic in a private university hospital in Metro Manila.

Resident physicians in the Department of Pathology used the technique prescribed by the Scandinavian pioneers utilizing a 10 -mL syringe with $23 \mathrm{G}$ to $25 \mathrm{G}$ needles attached to a syringe holder. Some surgeons and endocrinologists performed the procedure without a syringe holder. Smears were prepared by the aspirationist and cytotechnicians stained the smears using either Papanicolaou stain or modified Wright-Giemsa stain (Diff-Quik), or both. All interpretations were rendered by the pathologist on duty.

In the 1990s, private hospitals in the national capital region (Metro Manila) likewise engaged in the practice of thyroid FNA with pathologists, surgeons, and endocrinologists performing the procedure. Pathologists who were trained from the university 
hospital performed thyroid FNA in their affiliated hospitals when they started their practice in various regions of the country.

In 2010, interventional radiologists, endocrinologists, and cytopathologists started doing thyroid FNA under ultrasound-guidance, especially for small lesions measuring from 1.0 to $1.5 \mathrm{~cm}$.

Local endocrinologists follow the 2015 guidelines of the American Thyroid Association. In 2011, a revisit of the clinical practice guidelines at the Philippine General Hospital for patients with thyroid cancer maintained the original 2008 recommendation of total or near total thyroidectomy for all patients with well differentiated thyroid carcinoma with nodule size of greater than $1.0 \mathrm{~cm}$ in diameter, and lobectomy with an isolated nodule size of less than $1.0 \mathrm{~cm}$ in diameter, and without lymph node metastasis on preoperative ultrasound. ${ }^{8}$ There is no specific recommendation on preoperative FNA including terminology referable to TBSRTC. Intra-operative frozen section for non-diagnostic FNA is controversial as no consensus was reached by the multispecialty members of the panel. Likewise, international guidelines do not recommend the use of frozen section due to its limited role with high frequency of false-negative results and lack of consistent agreement between frozen section and final histological diagnosis. ${ }^{9}$

\section{TECHNIQUE OF FINE-NEEDLE ASPIRATION}

Most practicing pathologists performing thyroid FNA use a syringe holder made of aluminum or plastic. The procedure starts with proper positioning of the patient, application of $70 \%$ alcohol at the puncture site, and localization of the lesion by immobilizing the target with one hand followed by needle puncture of the skin into the target lesion. Negative pressure or suction is applied by back and forth cutting motions within the thyroid nodule and releasing this negative pressure prior to withdrawal of the needle once the material is obtained. Some surgeons and endocrinologists use only a syringe without a syringe holder while others use the non-aspirating technique. ${ }^{10}$

\section{PREPARATION OF SAMPLE}

The physician-aspirationist prepares the smears by placing a small drop of the aspirated material onto a glass slide. Smear is made by laying another glass on top of the sample material and pulling the slides apart to spread it. Wet smears are fixed in $95 \%$ ethyl alcohol or air dried for submission to the laboratory. Wet smears are subjected to Papanicolaou stain while air dried smears are prepared for modified Wright-Giemsa stain (Diff-Quik). The latter stain is also used in cases where there is an on-site request for rapid evaluation of the aspirates. All smears and liquid samples obtained from cyst fluids are processed in the laboratory by cytotechnicians. Smears are routinely stained with Papanicolaou stain and the remains of the samples are processed as cell block and stained with hematoxylin and eosin. Liquid based cytology is not used for thyroid aspirates.

\section{CYTOPATHOLOGY AND CYTOTECHNOLOGY TRAINING}

Formal cytopathology and cytotechnology training programs are not offered in the country at present. However, cytopathology is included as a rotation in anatomic pathology training programs. In both government and private hospitals with training in pathology, all aspirates are screened by pathology resident physician trainees and final cytodiagnosis is signed out by the pathologist on duty. In the absence of a training program, the pathologist on duty at the cytology unit screens and issues the final cytodiagnosis.

Pathologists interested in cytopathology have to study abroad for fellowship in cytopathology, after which they can apply to the local pathology society for recognition. To date, there are ten cytopathologists in the whole country, two of whom are certified by the local pathology society. Majority of the practice is in the national capital region.

There is no formal subspecialty society for cytopathology within the national organization of pathologists. Lectures in cytopathology are incorporated in the scientific program during the annual convention of the Philippine Society of Pathologists. Within individual training institutions, conferences in cytopathology are embedded in the training program of anatomic pathology.

\section{THYROID CYTOLOGY REPORTING SYSTEM}

Prior to the 2009 publication of TBSRTC, ${ }^{11}$ which is a standardized six-tier nomenclature, the aspiration biopsy cytology method was used., ${ }^{4,5}$ This method provides a spectrum of diagnosis that closely resembles the histopathologic diagnosis of thyroid disorders. In most instances, it is cytologically possible to categorize a thyroid lesion into one of the three main entities: hyperplasia/adenomatous colloid nodule, thyroiditis, and neoplasm. There are also indeterminate cases in which the presence of cellular atypia cannot totally rule out a malignancy. For this, "atypia suspicious for malignancy" cytodiagnosis is given and followed by a recommendation for clinical correlation or for fur- 
ther investigation if warranted. Cytodiagnosis is based on the assessment of cellularity, architectural or group patterns of diagnostic cells, individual cell cytology including nuclear and cytoplasmic characteristics, and the presence of other cells and materials in the background. Criteria for non-diagnostic aspirates (category I) follow the criteria prescribed by TBSRTC, and these include bloody samples, presence of blood and colloid only, paucicellular smears with less than six follicular cell groups of ten cells each, and poorly prepared smears. ${ }^{11}$ Aspiration performed with dissolution of the nodule is compatible with a thyroid cyst and is not considered as non-diagnostic if pathologist has a clear knowledge of the patient's biopsy findings. With the introduction of TBSRTC in 2009, a majority of the thyroid FNA reports utilized both descriptive diagnosis and its corresponding equivalent category number in the six-tier nomenclature for a more effective understanding by the referring physicians.

Difficult thyroid aspirates are handled in various ways by pathologists. Self-review is done with subsequent intradepartmental referral to another pathologist if a cytopathologist is not part of the hospital staff. Presently, the Philippines is still in the process of developing an external quality assurance program in cytopathology.

Based on a recent survey of FNA practice in 16 hospitals in the country, thyroid FNA comprised $46 \%$ to $85 \%$ of the total FNA in five major hospitals at the national capital region, and $30 \%$ to $85 \%$ in 11 hospitals in various regions of the country. At the Philippine General Hospital, 60\% of aspirates were from the thyroid. Being a training hospital, it has the highest number of non-diagnostic aspirates, ranging from $37 \%$ to $46 \%$ with an average of $42 \%$. The high number is unique since different resident physician trainees perform the procedure in the course of their rotation in the departments engaged in FNA. Four private hospitals from the national capital region with a bed capacity from 300 to almost 1,000 had a non-diagnostic yield ranging from $5 \%$ to $7 \%$. In the various regions of the country from north to south, category I registered 0 to $19 \%$ from a survey of four governments and seven private hospitals with a 300-400 bed capacity in the former and 90-680 bed capacity in the latter. Category I was $0 \%$ in one private hospital in southern Philippines since the physician-aspirationist is a pathologist who always makes a rapid on-site evaluation as part of FNA. Benign aspirates (category II) comprised $50 \%$ to $85 \%$. Aspirates in the category III accounted for $1 \%$ to $20 \%$ and were re-aspirated following the recommendation of TBSRTC. Category IV aspirates ranged from $1 \%$ to $14 \%$, category $\mathrm{V}$ from $1 \%$ to $10 \%$, and category VI from $2 \%$ to $20 \%$.

\section{THYROID CYTOLOGY AUDIT PROGRAM}

Patients nowadays are highly mobile and may seek medical services in different institutions. It often happens that FNA is done in one hospital and thyroid surgery with histopathologic diagnosis is performed in another center. However, all thyroid surgeries with previous FNA done in the same hospital are reviewed as part of the hospitals' monitoring program. No data has been released but may soon be forthcoming.

\section{STATUS OF ANCILLARY TESTING}

Ancillary studies of thyroid FNA are infrequently used. Immunohistochemistry in the diagnosis of thyroid tumors such as galectin-3, cytokeratin 19, and HBME-1 are only rarely utilized in a few hospitals at the national capital region for thyroid surgical pathology specimens and not in aspirates. Molecular testing for somatic mutations is yet to be developed because most of the patients opt thyroid surgery with just regular follow-up. ${ }^{12,13}$ Health care insurance does not cover molecular testing for somatic mutations. If requested, molecular testing is mainly physician driven. If the patient agrees to pay for the molecular testing, the test is referred to neighboring Asian countries or to North America.

\section{REVIEW OF PUBLICATIONS}

Researches on thyroid cytology in the country are mainly cytohistologic correlations with sensitivity, specificity, and accuracy rates. Cytohistologic correlation from three hospitals in the national capital region yielded a wide range of sensitivity from $30.7 \%$ to $73 \%$, specificity from $83 \%$ to $100 \%$, and accuracy from $72.8 \%$ to $87.2 \%{ }^{14-16}$ The low sensitivity can be attributed to poor tissue sampling since most thyroid FNAs are done by palpation only. A majority (85\%) of the discordant cases were due to sampling errors attributable to dual pathology with a dominant benign lesion, missing a small malignant focus which is usually a papillary microcarcinoma. ${ }^{14}$ The reliability can be improved if FNA is guided by ultrasound as attested in both international and local studies. ${ }^{16-18}$ Solid nodules with microcalcifications and irregular margins which were found to be significant predictors of malignancy can be readily detected. ${ }^{19,20}$ Improvement in diagnostic yield and accuracy was observed from $82.6 \%$ to $86.2 \%$ with ultrasound guidance. ${ }^{16}$ When combined with frozen section examination, ultrasound-guided FNA for concordant cases approached an accuracy of $97.2 \%$ in one study. ${ }^{21}$

The incidence of each diagnostic category of the Bethesda 
Table 1. Distribution of thyroid fine-needle aspiration diagnoses by the Bethesda system

\begin{tabular}{|c|c|c|c|c|c|c|c|c|c|}
\hline \multirow{2}{*}{ Study } & \multirow{2}{*}{ Hospital } & \multirow{2}{*}{ Year } & \multicolumn{6}{|c|}{ Distribution by the Bethesda categories (\%) } & \multirow{2}{*}{ Nodule } \\
\hline & & & I & $\|$ & III & IV & V & $\mathrm{Vl}$ & \\
\hline Young et al. (2011) ${ }^{24}$ & St. Luke's Medical Center, Quezon City & $2007-2009$ & 23.1 & 64.7 & & $9.4^{a}$ & & 2.8 & 2,239 \\
\hline Salillas and Almocera $(2016)^{25}$ & Private practice, Cebu City and Bohol & 2010-2014 & 1.3 & 56.2 & 5.4 & 11.1 & 13.2 & 12.8 & 3,799 \\
\hline
\end{tabular}

aSum of indeterminate categories III to V: classified as "indeterminate."

Table 2. Cytohistopathologic correlations in operated thyroid nodules

\begin{tabular}{|c|c|c|c|c|c|c|c|c|c|}
\hline \multirow{2}{*}{ Study } & \multirow{2}{*}{ Hospital } & \multirow{2}{*}{ Year } & \multicolumn{6}{|c|}{ Risk of malignancy for the Bethesda categories (\%) } & \multirow{2}{*}{ Nodule } \\
\hline & & & 1 & $\|$ & III & IV & V & $\mathrm{Vl}$ & \\
\hline Young et al. (2011) ${ }^{24}$ & St. Luke's Medical Center, Quezon City & $2007-2009$ & 17.2 & 9.6 & & $36.5^{\mathrm{a}}$ & & 76.5 & 251 \\
\hline Abelardo et al. $(2011)^{15}$ & Philippine General Hospital, Manila & 2010 & 44.4 & 18.9 & 40.0 & 44.4 & 60.0 & 100.0 & 99 \\
\hline Canete et al. $(2014)^{19}$ & Philippine General Hospital, Manila & 2008-2011 & $\mathrm{n} / \mathrm{a}$ & 32.0 & $\mathrm{n} / \mathrm{a}$ & 41.4 & 89.7 & 100.0 & 837 \\
\hline Carlos et al. (2014) & St. Luke's Medical Center, Quezon City & 2012-2013 & $\mathrm{n} / \mathrm{a}$ & $\mathrm{n} / \mathrm{a}$ & 35.3 & $\mathrm{n} / \mathrm{a}$ & $\mathrm{n} / \mathrm{a}$ & $\mathrm{n} / \mathrm{a}$ & 68 \\
\hline Ramos and Mirasol (2014) $)^{16}$ & St. Luke's Medical Center, Quezon City & 2012-2013 & 33.3 & 13.5 & 22.2 & 33.3 & 72.2 & 100.0 & 175 \\
\hline Salillas et al. $(2015)^{23}$ & SWU-MHAM College of Medicine, Cebu City & 2009-2012 & $n / a$ & 2.6 & 50.0 & 50.0 & 78.0 & 100.0 & 80 \\
\hline Salillas and Almocera $(2016)^{25}$ & Private practice, Cebu City and Bohol & 2010-2014 & $\mathrm{n} / \mathrm{a}$ & $\mathrm{n} / \mathrm{a}$ & 39.4 & $\mathrm{n} / \mathrm{a}$ & $\mathrm{n} / \mathrm{a}$ & $\mathrm{n} / \mathrm{a}$ & 33 \\
\hline Abelardo and Abesamis $(2016)^{14}$ & The Medical City, Pasig City & 2010-2015 & $\mathrm{n} / \mathrm{a}$ & 26.5 & $\mathrm{n} / \mathrm{a}$ & $\mathrm{n} / \mathrm{a}$ & \multicolumn{2}{|c|}{$71.4^{\mathrm{b}}$} & 448 \\
\hline \multicolumn{3}{|c|}{ The Bethesda system reference rate ${ }^{11}$} & $1-4$ & $0-3$ & $5-15$ & $15-30$ & $60-75$ & $97-99$ & \\
\hline
\end{tabular}

n/a, not available.

aSum of indeterminate categories III to V: classified as "indeterminate"; bOverall risk of malignancy for suspicious for malignancy and malignant cases.

system is rarely addressed in local studies (Table 1). Summary of available publications on the malignancy risk in the Bethesda categories is presented in Table 2. An overall risk of malignancy for malignant and suspicious for malignant cytodiagnosis is $71.4 \%$ while the overall risk of malignancy for benign cytodiagnosis is $26.5 \% .^{14}$ The risk of malignancy for category III ranges from 35.3\% to $50 \%,{ }^{15,22,23}$ which is higher than TBSRTC reference rate. Conveying these rates to our clinical colleagues with the recommendation to do a repeat FNA should be reconsidered for optimal patient care. 22,23

\section{FUTURE CHALLENGES}

Although thyroid FNA was introduced more than 30 years ago, a centralized database may be difficult to construct because the FNA practice in the Philippines is highly individualistic. If teaching and training of FNA are structured into the major training programs of physicians, a standardized FNA protocol, particularly in specimen sampling, can be improved. Looking forward, there is a need to establish a national registry from various hospitals and training institutions. Quality assurance and other related issues can be addressed through the establishment of a local society of cytopathologists in order to develop and improve the art and science of FNA. Finally, collaborative networking amongst pathologists, endocrinologists and surgeons is a strong impetus toward unified practice in the diagnosis of thyroid disor- ders using FNA.

\section{Conflicts of Interest}

No potential conflict of interest relevant to this article was reported.

\section{Acknowledgments}

The author wishes to acknowledge the contribution of the following pathologists from various regions of the Philippines who responded to the survey of FNA practice in the Philippines: Elizabeth Ann Alcazaren, Ma.Lourdes Goco, and Rolando Lopez (National Capital Region); Brenda Rosuman (Luzon); Jude Martin Cimafranca, Marvi Dulnuan, David Pedroza, Ansarie Salpin, Dianne Singson, and Walter Villanueva (Visayas); Rachel Alegata, Kenneth Crisostomo, Sherjan Kalim, and Gerard Lamayra (Mindanao).

\section{REFERENCES}

1. Diamantis A, Magiorkinis E, Koutselini H. Fine-needle aspiration (FNA) biopsy: historical aspects. Folia Histochem Cytobiol 2009; 47: 191-7.

2. Martin HE, Ellis EB. Biopsy by needle puncture and aspiration. Ann Surg 1930; 92: 169-81.

3. Wu M, Burstein DE. Fine needle aspiration. Cancer Invest 2004; 22: 620-8. 
4. Linsk JA, Franzen S. Clinical aspiration cytology. Philadelphia: Lippincott, 1983.

5. Zajicek J. Aspiration biopsy cytology, Part I. Cytology of supradiaphragmatic organs. Basel: S. Karger, 1974.

6. Zajicek J. Aspiration biopsy cytology, Part II. Cytology of infradiaphragmatic organs. Basel: S. Karger, 1979.

7. Haugen BR, Alexander EK, Bible KC, et al. 2015 American Thyroid Association management guidelines for adult patients with thyroid nodules and differentiated thyroid cancer: the American Thyroid Association Guidelines Task Force on Thyroid Nodules and Differentiated Thyroid Cancer. Thyroid 2016; 26: 1-133.

8. Sison CM, Obaldo J, Matsuo J, Uy GL, Jaring C. University of the Philippines - Philippine General Hospital revised clinical practice guidelines for the management of well-differentiated thyroid carcinoma of follicular cell origin. J Asean Fed Endocr Soc 2012; 27: 4961.

9. Mulcahy MM, Cohen JI, Anderson PE, Ditamasso J, Schmidt W. Relative accuracy of fine-needle aspiration and frozen section in the diagnosis of well-differentiated thyroid cancer. Laryngoscope 1998; 108(4 Pt 1): 494-6.

10. Santos JE, Leiman G. Nonaspiration fine needle cytology: application of a new technique to nodular thyroid disease. Acta Cytol 1988; 32: 353-6.

11. Cibas ES, Ali SZ; NCI Thyroid FNA State of the Science Conference. The Bethesda System For Reporting Thyroid Cytopathology. Am J Clin Pathol 2009; 132: 658-65.

12. Bychkov A. Prevalence of BRAFV600E mutation in Asian patients with thyroid cancer. Malays J Pathol 2017; 39: 95-6.

13. Navarro-Locsin CG, Chang AM, Daroy ML, Alfon AC, Andal JJ, Padua PF. Clinical and histopathological profile of BRAF V600E mutation in conventional papillary thyroid carcinoma in a Filipino population. Malays J Pathol 2016; 38: 141-8.

14. Abelardo AD, Abesamis RG. Cytohistologic correlation of thyroid lesions: a six year review at the Medical City, Philippines. Acta Cytol 2016; 60(Suppl 1): 220.

15. Abelardo AD, Ang MA, Enriquez AM. Thyroid aspirates using the Bethesda System for Reporting Thyroid Cytopathology (TBSRTC) in the assessment of risk malignancy: a comparison with conventional system of cytodiagnosis. Proc Taiwan Soc Pathol 2011; 2: 148.

16. Ramos CO, Mirasol RC. Ultrasound guidance improves the diagnostic yield and accuracy of fine needle aspiration biopsy of thyroid nodules in detecting malignancy. J Thyroid Disorders Ther 2014; 3: 148.

17. Baloch ZW, LiVolsi VA. Fine-needle aspiration of thyroid nodules: past, present, and future. Endocr Pract 2004; 10: 234-41.

18. Lo TE, Uy AT, Maningat PD. Well-differentiated thyroid cancer: the Philippine General Hospital experience. Endocrinol Metab (Seoul) 2016; 31: 72-9.

19. Canete EJ, Sison-Pena CM, Jimeno CA. Clinicopathological, biochemical, and sonographic features of thyroid nodule predictive of malignancy among adult Filipino patients in a tertiary hospital in the Philippines. Endocrinol Metab (Seoul) 2014; 29: 489-97.

20. Puno-Ramos MP, Villa ML, Kasal RG, Arzadon J, Alcazaren EA. Ultrasound features of thyroid nodules predictive of thyroid malignancy as determined by fine needle aspiration biopsy. Philipp J Intern Med 2015; 53: 1-8.

21. Young J, Lumapas-Gonzalez CG, Mirasol R. The diagnostic accuracy of ultrasound guided fine-needle aspiration biopsy and intraoperative frozen section examination in nodular thyroid disease. J Asean Fed Endocr Soc 2011; 26: 44-50.

22. Carlos AD, Mirasol R, Thomas Aquino E, Lourdes Goco M, Rizelle Toledo P, Carl Santos K. Management and malignancy rate of thyroid nodules with a cytologic diagnosis of atypia or follicular lesion of undetermined significance. J Asean Fed Endocr Soc 2014; 29: 78-84.

23. Salillas AL, Sun FC, Almocera EG. Review of the Bethesda System for Reporting Thyroid Cytopathology: a local study in Bohol Island, Philippines. Acta Cytol 2015; 59: 77-82.

24. Young JK, Lumapas CG, Mirasol R. Sonographically guided fineneedle aspiration biopsy of thyroid nodules: correlation between cytologic and histopathologic findings. Philipp J Intern Med 2011; 49: 8-14.

25. Salillas AL, Almocera EG. The implication of atypia of undetermined significance (AUS) category to a pathologist in a third world country. Acta Cytol 2016; 60(Suppl 1): 50-1. 